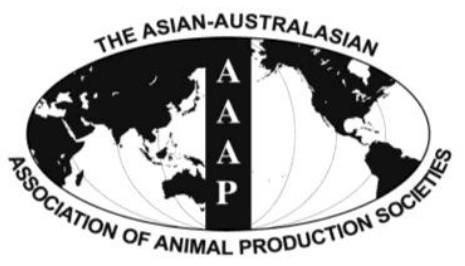

Asian-Aust. J. Anim. Sci.

Vol. 25, No. 9 : 1329 - 1337

September 2012

www.ajas.info

http://dx.doi.org/10.5713/ajas.2012.12125

\title{
Effects of Purple-fleshed Sweet Potato (Ipomoera batatas Cultivar Ayamurasaki) Powder Addition on Color and Texture Properties and Sensory Characteristics of Cooked Pork Sausages during Storage
}

\author{
Sang-Keun Jin, Yeong-Jung Kim, Jae Hong Park ${ }^{1}$, In-Chul Hur, Sang-Hae Nam ${ }^{2}$ and Daekeun Shin ${ }^{1,3, *}$ \\ Department of Animal Resources Technology, Gyeongnam National University of Science and Technology, \\ Jinju 660-758, Korea
}

\begin{abstract}
This study was conducted to evaluate the effects of adding purple-fleshed sweet potato (PFP) powder on the texture properties and sensory characteristics of cooked pork sausage. Sodium nitrite alone and sodium nitrite in combination with PFP were added to five different treatments sausages $(\mathrm{CON}$ (control) $=0.01 \%$ sodium nitrite, $\mathrm{SP} 25=0.005 \%$ sodium nitrite and $0.25 \%$ purplefleshed sweet potato powder combination, $\mathrm{SP} 50=0.005 \%$ sodium nitrite and $0.5 \%$ purple-fleshed sweet potato powder combination, PP25 $=0.25 \%$ purple-fleshed sweet potato powder, PP50 $=0.5 \%$ purple-fleshed sweet potato powder). The sausages were cooked to $74^{\circ} \mathrm{C}$, stored at $4^{\circ} \mathrm{C}$ for $6 \mathrm{wks}$, and used for chemical analysis, textural properties, and a sensory evaluation on $0,2,4$ and 6 wks of storage, respectively. Similar CIE a* and $\mathrm{b}^{*}$ values were determined in sausages from CON, SP25 and SP50 at the end of storage, and they were higher in CIE $a^{*}$ but lower in CIE $b^{*}$ than that of the PP25 and PP50 sausages. Significant differences were observed for brittleness and hardness when PFP was added to the sausages but were not confirmed after 4 wks of storage. The objective color score was influenced by adding PFP; however, the effect was not dose dependent. In overall acceptability, panelists favored the CON, SP25, SP50, and PP50 sausages but did not prefer PP25 sausages at the end of storage. Therefore, adding PFP to cooked pork sausages improved color and texture properties and sensory characteristics, but further study is needed to determine the proper ratio of sodium nitrite and PFP. (Key Words: Purple-fleshed Sweet Potato, Sodium Nitrite, Texture, Sensory Evaluation, Sausage)
\end{abstract}

\section{INTRODUCTION}

Due to the effects of nitrite/nitrate on sausage color, nitrite is a favorable additive for sausage dough. However, many countries in Europe, North America, and Asia limit the residual nitrite in sausage due to its effects on human health (Bozkurt and Erkmen, 2004). High health risks to humans due to adding nitrite include allergenic effects, vasodilator effects, and carcinogenic nitrosamine production (Marco et al., 2006). N-nitrosodimethylamine,

\footnotetext{
* Corresponding Author: Daekeun Shin. Tel: +82-55-751-3584, Fax: +82-55-751-3689, E-mail: aceflavor@hotmail.com

${ }^{1}$ Swine Science and Technology Center, Gyeongnam National University of Science and Technology, Jinju 660-758, Korea.

${ }^{2}$ Division of Food Science, Gyeongnam National University of Science and Technology, Jinju 660-758, Korea.

${ }^{3}$ Department of Animal Science, Chonbuk National University, Jeonju 561-756, Korea.

Submitted Mar. 6, 2012; Accepted May 29, 2012; Revised Jul. 1, 2012
}

$\mathrm{N}$-nitrosopyrrolidine, and N-nitrosopiperidine are the major carcinogenic compounds developed from nitrite in sausage (Ahn et al., 2002; Jo et al., 2003). Adding nitrite to sausage is not the only source of $\mathrm{N}$-nitrosamines, a very potent carcinogen, but $\mathrm{N}$-nitrosamines can be generated due to the process of smoking, salting, and pickling sausage (Ahn et al., 2002).

Adding nitrite to sausages forms $\mathrm{N}$-nitrosamines in meat products, thereby, many studies have shown that nitrosamines can be minimized and/or impeded, as nitrite ions are fully scavenged (Rundlöf et al., 2000; Lee et al., 2006). Many intrinsic and/or extrinsic factors enhance nitrite ion development in sausage, indicating that reducing nitrite is a valuable approach to lower $\mathrm{N}$-nitrosamine formation in meat products. The sweet potato (Ipomoea batatas L.) is rich in anthocyanins, vitamins, and minerals (Islam et al., 2002; Teow et al., 2007). Typically, purplefleshed sweet potato (PFP), which include 0.4 to $0.6 \mathrm{mg}$ anthocyanin/g fresh weight, mainly consists of mono- or di- 
acylated forms of cyanidin and peonidin (Hagiwara et al., 2002; Suda et al., 2003) and show high stability during cooking (Suda et al., 2003; Kano et al., 2005). In a study by Cevallos-Casals and Cisneros-Zevallos (2004), anthocyanin pigments tended to be influenced by $\mathrm{pH}$. The color stability of red sweet potatoes was secured at $\mathrm{pH} 0.9$ to 4 when they were stored at $20^{\circ} \mathrm{C}$ for $138 \mathrm{~d}$.

Anthocyanins extracted from either purple-fleshed or red sweet potato can be a color pigment, generating redviolet color in foods; however, only limited information is available when PFP powder is added to cooked pork sausages. The starch released from sweet potatoes by adding PFP powder to cooked pork sausages may influence textural properties and sensory characteristics. Therefore, our study was not only aimed at diminishing nitrite in cooked pork sausages by replacing nitrite with PFP powder but to provide information related to texture properties and sensory characteristics of cooked pork sausages with added PFP powder as a naturally derived partial nitrite replacer.

\section{MATERIALS AND METHODS}

\section{Sample preparation}

Approximately $23 \mathrm{~kg}$ of commercial pork loin meat was obtained from a local commercial store, and visible pork loin fat was removed. All pork loins were coarse ground (MGB-32, Hankook Fujee Industries Co. Ltd., Suwon, Republic of Korea) and weighed to allot pork loins into five different groups (4 kg/group).

\section{Preparation of cooked pork sausage}

Individual pork loin meat from each group was mixed as shown in Table 1 and ground to a fine texture. Nitrite and/or PFP were added to the mixture as follows: i) $0.01 \%$ nitrite (sodium nitrite, Duksan Pure Chemical Co. Ltd., Ansan, Republic of Korea; control), ii) $0.005 \%$ nitrite and $0.25 \%$ PFP powder (Jisanfood, Hamyang, Republic of Korea) combination, iii) $0.005 \%$ nitrite and $0.5 \%$ PFP combination, and iv) $0.25 \%$ PFP powder and v) $0.5 \%$ PFP powder. Mixing was performed for $2 \mathrm{~min}$ to achieve uniform distribution of the nitrite and/or PFP powder in the mixture matrix (A-20, Ramon Co. Ltd., Oiartzun, Spain). Approximately $160 \mathrm{~g}$ of mixture was used to stuff the sausages, (E-25, Hankook Fujee Industries Co. Ltd., Suwon, Republic of Korea), and $12 \mathrm{~cm}$ sausages were formed (Krehalon UK Ltd., East Riding of Yorkshire, UK). All sausages were placed in a water bath (BS-31, Jeio Tech. Co. Ltd., Seoul, Republic of Korea), uniformly cooked at $100^{\circ} \mathrm{C}$, removed when internal temperature reached $74^{\circ} \mathrm{C}$, and cooled at $4^{\circ} \mathrm{C}$ in a refrigerator for $1 \mathrm{~h}$. Each sausage was subsequently packed and covered with a polyvinyl chloride film (Krehalon UK Ltd., East Riding of Yorkshire, UK). All sausages from the five different treatments were stored at $4^{\circ} \mathrm{C}$ for chemical analysis and for sensory evaluations at weeks $0,2,4$, and 6 of storage. All materials to prepare the sausages were used on three different processing days for experimental replication.

\section{Moisture and crude protein content measurement}

Moisture and crude protein contents were determined as described by AOAC (1990). Moisture was determined by an oven-drying method described in \#934.01 and then expressed as percent (\%). The Kjeldhal method (\#984.13)

Table 1. Composition of cooked pork sausage blends ${ }^{1}$

\begin{tabular}{|c|c|c|c|c|c|}
\hline \multirow{2}{*}{ Ingredients } & \multicolumn{5}{|c|}{ Composition (\%) } \\
\hline & $\mathrm{CON}$ & SP25 & SP50 & PP25 & PP50 \\
\hline \multicolumn{6}{|l|}{ Meat ingredients } \\
\hline Pork loin meat & 72.40 & 72.40 & 72.4 & 72.4 & 72.4 \\
\hline Pork fat & 11.20 & 11.20 & 11.2 & 11.2 & 11.2 \\
\hline \multicolumn{6}{|l|}{ Nonmeat ingredients } \\
\hline Ice & 13.80 & 13.55 & 13.30 & 13.55 & 13.30 \\
\hline Salt $^{2}$ & 1.39 & 1.39 & 1.39 & 1.39 & 1.39 \\
\hline Sodium nitrite $\left(\mathrm{NaNO}_{2}\right)^{3}$ & 0.01 & 0.005 & 0.005 & 0.00 & 0.00 \\
\hline Phosphate $^{4}$ & 0.24 & 0.24 & 0.24 & 0.24 & 0.24 \\
\hline Sugar $^{5}$ & 0.50 & 0.50 & 0.50 & 0.50 & 0.50 \\
\hline $\mathrm{MSG}^{6}$ & 0.06 & 0.06 & 0.06 & 0.06 & 0.06 \\
\hline Spice $^{7}$ & 0.40 & 0.40 & 0.40 & 0.40 & 0.40 \\
\hline Purple-fleshed sweet potato powder & 0.00 & 0.25 & 0.50 & 0.25 & 0.50 \\
\hline Total & 100.00 & 100.00 & 100.00 & 100.00 & 100.00 \\
\hline
\end{tabular}

${ }^{1}$ Treatment: CON (control) $=0.01 \%$ sodium nitrite, SP25 $=0.005 \%$ sodium nitrite and $0.25 \%$ purple-fleshed sweet potato powder combination, SP50 $=$ $0.005 \%$ sodium nitrite and $0.5 \%$ purple-fleshed sweet potato powder combination, PP $25=0.25 \%$ purple-fleshed sweet potato powder, PP50 $=0.5 \%$ purple-fleshed sweet potato powder.

${ }^{2}$ Refined salt (Woo-Il S\&F Co., Ulsan, Republic of Korea). ${ }^{3}$ Sodium nitrite (Duksan Co., Kyungkido, Republic of Korea).

${ }^{4}$ FOS/ENR (Taewon Food Co., Kyungkido, Republic of Korea). ${ }^{5}$ Beksul white sugar (CJ cheiljedang Co., Incheon, Republic of Korea).

${ }^{6}$ Monosodium L-Glutanate (Shinwon Chemical Co. Ltd., Seoul, Republic of Korea). ${ }^{7}$ Bologna (Taewon Food Co., Kyungkido, Republic of Korea). 
was used for crude protein determination. Approximately 1 $\mathrm{g}$ of ground sausage was weighed, added to a digestion flask, and a series of sample digestions and distillations was conducted. Each sample was then titrated with sodium hydroxide and expressed as a percent (\%).

\section{pH, volatile basic nitrogen (VBN), and residual nitrite measurement}

To determine the $\mathrm{pH}$ value of the cooked pork sausage, $10 \mathrm{~g}$ of cooked pork sausages was homogenized with $90 \mathrm{ml}$ of double distilled water (DDW) for $20 \mathrm{~s}$ at 13,500 rpm (T25B, IKA Sdn. Bhd., Malaysia) and then a digital pH meter (Seven Easy pH, Mettler-Toledo AG, Schwerzenbach, Switzerland) was used. Prior to reading the $\mathrm{pH}$ value, the $\mathrm{pH}$ meter was calibrated with standard buffers at $\mathrm{pH} 4.0$ and 7.0, and duplicate readings per sample were obtained.

VBN was determined according to the Conway micropipette diffusion method modified by Pearson (1976) and expressed as mg VBN/100 $\mathrm{g}$ of sample. Briefly, $1 \mathrm{~g}$ of sample and a few drops of phenolphthalein indicator were mixed with $3.5 \mathrm{ml}$ of $20 \%$ sodium hydroxide solution in a distillated flask. The apparatus was sealed, and steam distillate was collected in a flask containing $20 \mathrm{ml}$ of $4 \%$ boric acid and a few drops of methyl red and methylene blue (2:1 mixture). The steam distillate was continuously collected until a $250 \mathrm{ml}$ distillated flask was filled. The solution obtained was then titrated using $0.01 \mathrm{M}$ hydrochloric acid, and titration was stopped when the green color changed to gray. The final VBN calculation was accomplished based on a VBN value from a blank containing $6 \%$ perchloric acid steam distillation.

Residual nitrite was determined using a spectrophotometric method as described in method \#973.31AOAC (1990). Briefly, 5 g of cooked pork sausage was mixed with $300 \mathrm{ml}$ of boiled water. Each sample was heated to $100^{\circ} \mathrm{C}$ in a water bath (BS-31, Jeio Tech. Co. Ltd., Seoul, Republic of Korea) for $2 \mathrm{~h}$. All samples were then filled to $500 \mathrm{ml}$ with hot water, filtered, and then transferred to a $50 \mathrm{ml}$ volumetric flask. A series of sulfanilamide (SPA) and N-ethylenediamine dihydrochloride (NED) additions were performed, and the final absorbance was measured at $540 \mathrm{~nm}$ against a blank containing $45 \mathrm{ml}$ DDW, $2.5 \mathrm{ml} \mathrm{SPA}$ and $2.5 \mathrm{ml} \mathrm{NED,} \mathrm{respectively.}$

\section{Color CIE $\mathrm{L}^{*}, \mathrm{a}^{*}$ and $\mathrm{b}^{*}$, whiteness, chroma, and hue measurement}

CIE L* (lightness), a* (redness), and $\mathrm{b}^{*}$ (yellowness) color space values as well as chroma (c) and hue (h) were determined using a Minolta colorimeter (Minolta Chroma Meter CR-300, Minolta Co., Ltd., Ramsey, NJ, USA). Before conducting each measurement, the colorimeter was calibrated daily $(\mathrm{Y}=92.8, \mathrm{x}=0.3134$ and $\mathrm{y}=0.3193)$, and two different readings per sample were acquired. All values from each sample were averaged and expressed as CIE L*, $a^{*}$, and $b^{*}$ color space values, chroma (c) and hue (h), respectively. Whiteness (W) was determined using the following formula; $\mathrm{W}=\mathrm{L}^{*}-3 \mathrm{~b}^{*}$.

\section{Shear force and textural property measurement}

Five sample pieces shaped to $1.5 \times 1.5 \times 1.5 \mathrm{~cm}$, were tempered at room temperature for $30 \mathrm{~min}$ to measure the shear force values of the cooked pork sausage. After equilibrating the sausage samples at room temperature, each sample was sheared once using an Instron 3343 (US/MX50, A\&D Co., USA) equipped with a Warner Bratzler shearing device providing a $100 \mathrm{~mm} / \mathrm{min}$ crosshead speed. The average shear force value from each treatment was calculated and expressed as $\mathrm{kg} / \mathrm{cm}^{2}$.

Textural properties were assessed using the modified method of Kealy (2006). All samples were equilibrated and processed at room temperature. To assess brittleness, hardness, cohesiveness, springiness, gumminess, chewiness, and adhesiveness, five piece cubes $(1.5 \times 1.5 \times 1.5 \mathrm{~cm})$ sampled from each treatment were subjected to a texture analyzer (EZ-test, Shimadzu, Tokyo, Japan) equipped with a cylindrically shaped plunger (5-mm diameter) and a 500-N load cell. The crosshead speed of the texture analyzer was $66 \mathrm{~mm} / \mathrm{min}$

\section{Sensory evaluation}

A sensory evaluation was performed at the $2 \mathrm{wk}$ interval during the 6 wks of storage. Seven panelists (age range, 25 to $40 \mathrm{yrs}$ ) participated, and they were trained based on the procedures outlined by AMSA (1995) and Meilgaard et al. (1991). All cooked pork sausage from each treatment was re-heated to $100^{\circ} \mathrm{C}$ for $5 \mathrm{~min}$ (Euro-Grill Tg101, Fri-Jad BV., Etten-Leur, The Netherlands) and cooled in a $35^{\circ} \mathrm{C}$ incubator (JS-IN-180, Johnsam Co., Seoul, Republic of Korea) for $1 \mathrm{~h}$. Each sausage sample was then cut into a 1.5 $\times 1.5 \times 1.5 \mathrm{~cm}$ cube and served to panelists with a three digit number. The color appearance, aroma, flavor, springiness, juiciness, and overall acceptability were evaluated based on a 9-point scale, $0=$ extremely dislike and $9=$ extremely like. Saltine crackers and unsalted DDW were served. Two different sessions with a 10 min break between sessions were conducted with three treatment samples per session containing a control sample.

\section{Statistical analysis}

Data from six sausage samples per treatment $(2 \times 6 \times 5=$ replication $\times$ sample $\times$ treatment) in duplicate were collected. All data collected by a one-way analysis of variance, treatment or storage day, respectively, and the Duncan's multiple range test using the SAS (1998) version 6.12 program (SAS Institute, Cary, NC, USA) Treatment effects 
were considered significantly different at $\mathrm{p}<0.05$.

\section{RESULTS}

\section{Moisture and crude protein content of cooked pork sausages}

An overall summary of moisture and crude protein content of the cooked pork sausages is provided in Table 2. Moisture did not differ significantly, but significant differences were observed for crude protein at week 0 of storage $(\mathrm{p}<0.05)$. Sausages sampled from both the SP25 and PP50 treatments showed the highest crude protein content at week 0 of storage $(\mathrm{p}<0.05)$; however, a similar trend was not measured on wk 6 of storage ( $p>0.05)$.

\section{pH, VBN, and residual nitrite measurement of cooked pork sausages}

Hydrogen ion concentration - $\mathrm{pH}$ values of 6.15 to 6.45 were determined for all treatments at week 0 of storage, and they decreased to 5.76 to 5.86 at 6 wks (Table 3). The highest $\mathrm{pH}$ with a significant difference was measured in sausages containing $0.01 \%$ nitrite only, whereas the PP25 or PP50 sausages had the lowest $\mathrm{pH}$ values during storage $(\mathrm{p}<0.05)$

VBNs were not significantly influenced by adding PFP powder (Table 3). However, sausages containing PFP powder stored for 6 weeks released less volatile nitrogen than that of control sausages $(24.55 \pm 3.39)(\mathrm{p}<0.05)$.

As expected, the highest amount of nitrite remained
Table 2. Proximate composition of cooked pork sausages containing two different percentages of purple sweet potato combined with nitrite during 6 wks of storage at $4^{\circ} \mathrm{C}$

\begin{tabular}{llcc}
\hline \multirow{2}{*}{ Items } & Treatments $^{1}$ & \multicolumn{2}{c}{ Storage (wks) } \\
\cline { 3 - 4 } & & 0 & 6 \\
\hline Moisture & CON & $67.42 \pm 0.79$ & $67.73 \pm 0.02$ \\
& SP25 & $67.77 \pm 0.02$ & $67.79 \pm 0.06$ \\
& SP50 & $67.57 \pm 0.08$ & $67.51 \pm 0.42$ \\
& PP25 & $67.51 \pm 0.42$ & $67.94 \pm 0.00$ \\
Crude protein & PP50 & $67.69 \pm 0.05$ & $67.71 \pm 0.05$ \\
& CON & $20.55 \pm 0.15^{\mathrm{b}}$ & $19.74 \pm 0.10$ \\
& SP25 & $22.95 \pm 0.05^{\mathrm{a}}$ & $20.49 \pm 0.13$ \\
& SP50 & $19.53 \pm 0.08^{\mathrm{c}}$ & $20.81 \pm 0.06$ \\
& PP25 & $19.72 \pm 0.52^{\mathrm{c}}$ & $20.56 \pm 1.80$ \\
& PP50 & $22.95 \pm 0.24^{\mathrm{a}}$ & $19.33 \pm 0.21$ \\
\hline
\end{tabular}

1 Treatment: CON (control) $=0.01 \%$ sodium nitrite, $\mathrm{SP} 25=0.005 \%$ sodium nitrite and $0.25 \%$ purple-fleshed sweet potato powder combination, SP50 $=0.005 \%$ sodium nitrite and $0.5 \%$ purple-fleshed sweet potato powder combination, PP25 $=0.25 \%$ purple-fleshed sweet potato powder, PP50 $=0.5 \%$ purple-fleshed sweet potato powder.

a,b,c Mean values within a column followed by the same letter are not significantly different $(\mathrm{p}>0.05)$

when $0.01 \%$ nitrite was added to sausages $(\mathrm{CON})$ when compared to sausages from other treatments (Table 3). Additionally, more residual nitrite was measured among sausages in the SP25 and SP50 treatments than sausages in the PP25 and PP50 treatments during the 6 wks of storage. Adding PFP powder to the sausages tended to result in

Table 3. Hydrogen ion concentration $(\mathrm{pH})$, volatile basic nitrogen (VBN), and residual nitrite of cooked pork sausages containing two different percentages of purple sweet potato combined with nitrite during $6 \mathrm{wks}$ of storage at $4^{\circ} \mathrm{C}$

\begin{tabular}{llcccc}
\hline \multirow{2}{*}{ Items } & Treatments $^{1}$ & \multicolumn{4}{c}{ Storage (wks) } \\
\cline { 3 - 6 } $\mathrm{pH}$ & CON & $6.45 \pm 0.01^{\mathrm{a}}$ & $6.29 \pm 0.02^{\mathrm{a}}$ & $6.10 \pm 0.02^{\mathrm{a}}$ & $5.86 \pm 0.01^{\mathrm{a}}$ \\
& SP25 & $6.20 \pm 0.01^{\mathrm{c}}$ & $6.03 \pm 0.00^{\mathrm{d}}$ & $5.77 \pm 0.02^{\mathrm{c}}$ & $5.81 \pm 0.01^{\mathrm{c}}$ \\
& SP50 & $6.26 \pm 0.02^{\mathrm{b}}$ & $6.09 \pm 0.00^{\mathrm{b}}$ & $5.87 \pm 0.01^{\mathrm{b}}$ & $5.84 \pm 0.01^{\mathrm{b}}$ \\
& PP25 & $6.17 \pm 0.00^{\mathrm{d}}$ & $5.99 \pm 0.00^{\mathrm{e}}$ & $5.74 \pm 0.01^{\mathrm{d}}$ & $5.80 \pm 0.01^{\mathrm{c}}$ \\
& PP50 & $6.15 \pm 0.01^{\mathrm{d}}$ & $6.06 \pm 0.01^{\mathrm{c}}$ & $5.78 \pm 0.01^{\mathrm{c}}$ & $5.76 \pm 0.02^{\mathrm{d}}$ \\
VBN & CON & $11.48 \pm 0.28$ & $16.94 \pm 3.61$ & $16.53 \pm 0.84$ & $24.55 \pm 3.39^{\mathrm{a}}$ \\
$(\mathrm{mg}$ \%) & SP25 & $11.71 \pm 0.08$ & $16.24 \pm 1.27$ & $17.12 \pm 0.58$ & $21.28 \pm 2.22^{\mathrm{ab}}$ \\
& SP50 & $11.39 \pm 0.32$ & $14.75 \pm 1.98$ & $16.19 \pm 1.01$ & $19.69 \pm 0.32^{\mathrm{b}}$ \\
Residual nitrite & PP25 & $11.29 \pm 0.58$ & $16.94 \pm 1.17$ & $18.73 \pm 2.14$ & $19.88 \pm 0.40^{\mathrm{b}}$ \\
& PP50 & $11.85 \pm 0.16$ & $14.89 \pm 1.56$ & $15.44 \pm 0.32$ & $17.22 \pm 0.59^{\mathrm{b}}$ \\
& CON & $7.10 \pm 0.77^{\mathrm{a}}$ & $5.49 \pm 0.13^{\mathrm{a}}$ & $4.57 \pm 0.06^{\mathrm{a}}$ & $3.38 \pm 0.06^{\mathrm{a}}$ \\
& SP25 & $4.09 \pm 0.41^{\mathrm{b}}$ & $2.96 \pm 0.02^{\mathrm{c}}$ & $2.28 \pm 0.05^{\mathrm{c}}$ & $1.76 \pm 0.05^{\mathrm{c}}$ \\
& SP50 & $4.12 \pm 0.25^{\mathrm{b}}$ & $3.12 \pm 0.02^{\mathrm{b}}$ & $2.46 \pm 0.06^{\mathrm{b}}$ & $1.85 \pm 0.05^{\mathrm{b}}$ \\
& PP25 & $1.40 \pm 0.16^{\mathrm{c}}$ & $0.12 \pm 0.02^{\mathrm{d}}$ & $0.03 \pm 0.00^{\mathrm{d}}$ & $0.04 \pm 0.01^{\mathrm{d}}$ \\
& PP50 & $1.53 \pm 0.13^{\mathrm{c}}$ & $0.11 \pm 0.01^{\mathrm{d}}$ & $0.05 \pm 0.01^{\mathrm{d}}$ & $0.05 \pm 0.00^{\mathrm{d}}$ \\
\hline
\end{tabular}

Data represent mean \pm standard deviation.

${ }^{1}$ Treatment: CON (control) $=0.01 \%$ sodium nitrite, SP25 $=0.005 \%$ sodium nitrite and $0.25 \%$ purple-fleshed sweet potato powder combination, SP50 $=$ $0.005 \%$ sodium nitrite and $0.5 \%$ purple-fleshed sweet potato powder combination, PP25 $=0.25 \%$ purple-fleshed sweet potato powder, PP50 $=0.5 \%$ purple-fleshed sweet potato powder.

a,b,c,d Mean values within a column followed by the same letter are not significantly different $(\mathrm{p}>0.05)$. 
significantly higher amounts of residual nitrite when PFP powder was combined with sodium nitrite and then added to the sausages.

CIE L*, a* and $\mathbf{b}^{*}$, whiteness (W), chroma (c) and hue (H) measurement of cooked pork sausages

Color was assessed to evaluate the effects of adding PFP powder on cooked pork sausages (Table 4). Significantly higher lightness was observed at up to 2 wks of storage in sausages from both the control and PP25 treatments $(\mathrm{p}<0.05)$, but the highest lightness was maintained at $6 \mathrm{wks}$ of storage when $0.25 \%$ PFP powder was added to the sausages $(\mathrm{p}<0.05)$. However, the redness of the PP25 and PP50 sausages was not high enough to compare to the red color of sausages from other treatments during the 6 wks of storage. Sausages in both the SP25 and SP50 groups had low redness values at 0 wks of storage but showed similar redness at 4 and/or 6 wks of storage compared to that of control sausages. High yellowness values were obtained when only PFP powder was added to the sausages and then stored for up to $6 \mathrm{wks}$.

The whiteness, chroma, and hue values of sausages were influenced by adding sodium nitrite and PFP powder to the sausages. Adding sodium nitrite positively affected sausage whiteness; however, negative effects on the chroma and hue values were observed when PFP powder was added, and the sausages were stored for up to $6 \mathrm{wks}$. Thus, the PP25 and PP50 sausages showed the lowest whiteness but

Table 4. CIE L*, $a^{*}$ and $b^{*}$, whiteness $(\mathrm{W})$, chroma (c), hue $(\mathrm{H})$ and residual nitrite content of cooked pork sausages containing two different percentages of purple sweet potato combined with nitrite during 6 wks of storage at $4^{\circ} \mathrm{C}$

\begin{tabular}{|c|c|c|c|c|c|}
\hline \multirow{2}{*}{ Items } & \multirow{2}{*}{ Treatments $^{1}$} & \multicolumn{4}{|c|}{ Storage (wks) } \\
\hline & & 0 & 2 & 4 & 6 \\
\hline \multirow[t]{5}{*}{$\mathrm{L}^{*}$} & $\mathrm{CON}$ & $79.52 \pm 0.18^{\mathrm{a}}$ & $79.72 \pm 0.05^{\mathrm{a}}$ & $79.92 \pm 0.32^{\mathrm{b}}$ & $79.52 \pm 0.22^{\mathrm{b}}$ \\
\hline & SP25 & $77.65 \pm 0.22^{\mathrm{b}}$ & $77.48 \pm 0.75^{\mathrm{c}}$ & $77.63 \pm 0.11^{\mathrm{d}}$ & $78.06 \pm 0.47^{\mathrm{d}}$ \\
\hline & SP50 & $76.68 \pm 0.25^{\mathrm{c}}$ & $76.92 \pm 0.19^{c}$ & $76.83 \pm 0.02^{\mathrm{e}}$ & $76.99 \pm 0.20^{\mathrm{e}}$ \\
\hline & PP25 & $79.72 \pm 0.34^{\mathrm{a}}$ & $80.23 \pm 0.23^{\mathrm{a}}$ & $80.37 \pm 0.26^{\mathrm{a}}$ & $80.63 \pm 0.24^{\mathrm{a}}$ \\
\hline & PP50 & $78.02 \pm 0.16^{\mathrm{b}}$ & $78.74 \pm 0.40^{\mathrm{b}}$ & $78.67 \pm 0.17^{\mathrm{c}}$ & $78.92 \pm 0.12^{\mathrm{c}}$ \\
\hline \multirow[t]{5}{*}{$a^{*}$} & $\mathrm{CON}$ & $3.56 \pm 0.07^{\mathrm{a}}$ & $7.05 \pm 0.05^{\mathrm{b}}$ & $7.18 \pm 0.09^{\mathrm{a}}$ & $6.86 \pm 0.52^{\mathrm{a}}$ \\
\hline & SP25 & $2.26 \pm 0.09^{c}$ & $6.72 \pm 0.04^{\mathrm{b}}$ & $6.61 \pm 0.13^{\mathrm{b}}$ & $6.42 \pm 0.72^{\mathrm{a}}$ \\
\hline & SP50 & $3.18 \pm 0.12^{\mathrm{b}}$ & $7.50 \pm 0.33^{\mathrm{a}}$ & $7.41 \pm 0.13^{\mathrm{a}}$ & $6.86 \pm 0.14^{\mathrm{a}}$ \\
\hline & PP25 & $-1.23 \pm 0.13^{\mathrm{e}}$ & $1.76 \pm 0.11^{\mathrm{d}}$ & $1.34 \pm 0.07^{\mathrm{d}}$ & $1.68 \pm 0.32^{\mathrm{b}}$ \\
\hline & PP50 & $-0.35 \pm 0.05^{\mathrm{d}}$ & $2.88 \pm 0.27^{\mathrm{c}}$ & $2.35 \pm 0.30^{c}$ & $2.18 \pm 0.29^{\mathrm{b}}$ \\
\hline \multirow[t]{5}{*}{$b^{*}$} & $\mathrm{CON}$ & $11.40 \pm 0.12^{\mathrm{c}}$ & $7.61 \pm 0.06^{\mathrm{c}}$ & $7.43 \pm 0.04^{\mathrm{d}}$ & $7.89 \pm 0.45^{\mathrm{b}}$ \\
\hline & SP25 & $11.45 \pm 0.14^{\mathrm{c}}$ & $7.52 \pm 0.05^{\mathrm{c}}$ & $7.92 \pm 0.04^{c}$ & $7.93 \pm 0.42^{\mathrm{b}}$ \\
\hline & SP50 & $11.19 \pm 0.07^{\mathrm{c}}$ & $7.09 \pm 0.44^{\mathrm{d}}$ & $7.62 \pm 0.14^{\mathrm{d}}$ & $8.16 \pm 0.09^{b}$ \\
\hline & PP25 & $13.50 \pm 0.15^{\mathrm{a}}$ & $10.26 \pm 0.03^{\mathrm{a}}$ & $11.07 \pm 0.11^{\mathrm{a}}$ & $10.77 \pm 0.27^{\mathrm{a}}$ \\
\hline & PP50 & $12.85 \pm 0.20^{\mathrm{b}}$ & $9.53 \pm 0.09^{\mathrm{b}}$ & $10.34 \pm 0.15^{\mathrm{b}}$ & $10.54 \pm 0.16^{\mathrm{a}}$ \\
\hline \multirow[t]{5}{*}{ W } & $\mathrm{CON}$ & $45.33 \pm 0.52^{\mathrm{a}}$ & $56.88 \pm 0.20^{\mathrm{a}}$ & $57.63 \pm 0.38^{\mathrm{a}}$ & $55.86 \pm 1.46^{\mathrm{a}}$ \\
\hline & SP25 & $43.31 \pm 0.61^{\mathrm{b}}$ & $54.92 \pm 0.87^{\mathrm{b}}$ & $53.87 \pm 0.22^{\mathrm{b}}$ & $54.27 \pm 0.79^{\mathrm{b}}$ \\
\hline & SP50 & $43.12 \pm 0.16^{\mathrm{b}}$ & $55.65 \pm 1.40^{\mathrm{ab}}$ & $53.97 \pm 0.42^{\mathrm{b}}$ & $52.50 \pm 0.32^{\mathrm{c}}$ \\
\hline & PP25 & $39.21 \pm 0.79^{c}$ & $49.46 \pm 0.19^{c}$ & $47.16 \pm 0.37^{\mathrm{c}}$ & $48.31 \pm 0.56^{\mathrm{d}}$ \\
\hline & PP50 & $39.47 \pm 0.77^{\mathrm{c}}$ & $50.16 \pm 0.66^{\mathrm{c}}$ & $47.66 \pm 0.35^{\mathrm{c}}$ & $47.30 \pm 0.38^{\mathrm{d}}$ \\
\hline \multirow[t]{5}{*}{$\mathrm{c}$} & $\mathrm{CON}$ & $11.94 \pm 0.12^{\mathrm{c}}$ & $10.37 \pm 0.01^{\mathrm{a}}$ & $10.34 \pm 0.09^{c}$ & $10.47 \pm 0.07^{\mathrm{b}}$ \\
\hline & SP25 & $11.67 \pm 0.14^{\mathrm{d}}$ & $10.08 \pm 0.06^{\mathrm{b}}$ & $10.32 \pm 0.05^{\mathrm{c}}$ & $10.22 \pm 0.14^{\mathrm{c}}$ \\
\hline & SP50 & $11.63 \pm 0.04^{\mathrm{d}}$ & $10.33 \pm 0.06^{\mathrm{a}}$ & $10.62 \pm 0.10^{\mathrm{b}}$ & $10.67 \pm 0.04^{\mathrm{ab}}$ \\
\hline & PP25 & $13.56 \pm 0.15^{\mathrm{a}}$ & $10.41 \pm 0.01^{\mathrm{a}}$ & $11.15 \pm 0.10^{\mathrm{a}}$ & $10.91 \pm 0.23^{\mathrm{a}}$ \\
\hline & PP50 & $12.86 \pm 0.21^{\mathrm{b}}$ & $9.96 \pm 0.16^{\mathrm{b}}$ & $10.60 \pm 0.08^{\mathrm{b}}$ & $10.77 \pm 0.10^{\mathrm{a}}$ \\
\hline \multirow[t]{5}{*}{$\mathrm{H}$} & $\mathrm{CON}$ & $72.65 \pm 0.38^{\mathrm{e}}$ & $47.20 \pm 0.41^{\mathrm{c}}$ & $45.99 \pm 0.27^{\mathrm{d}}$ & $49.02 \pm 3.80^{\mathrm{b}}$ \\
\hline & SP25 & $78.85 \pm 0.40^{\mathrm{c}}$ & $48.22 \pm 0.04^{c}$ & $50.17 \pm 0.70^{c}$ & $51.07 \pm 4.57^{\mathrm{b}}$ \\
\hline & SP50 & $74.15 \pm 0.63^{\mathrm{d}}$ & $43.36 \pm 3.04^{\mathrm{d}}$ & $45.83 \pm 0.86^{\mathrm{d}}$ & $49.96 \pm 0.87^{b}$ \\
\hline & PP25 & $95.20 \pm 0.57^{\mathrm{a}}$ & $80.28 \pm 0.62^{\mathrm{a}}$ & $83.09 \pm 0.42^{\mathrm{a}}$ & $81.11 \pm 1.81^{\mathrm{a}}$ \\
\hline & PP50 & $91.58 \pm 0.20^{\mathrm{b}}$ & $73.18 \pm 1.31^{\mathrm{b}}$ & $77.21 \pm 1.74^{\mathrm{b}}$ & $78.33 \pm 1.65^{\mathrm{a}}$ \\
\hline
\end{tabular}

Data represent mean \pm standard deviation.

${ }^{1}$ Treatment: CON (control) $=0.01 \%$ sodium nitrite, SP25 $=0.005 \%$ sodium nitrite and $0.25 \%$ purple-fleshed sweet potato powder combination, SP50 $=$ $0.005 \%$ sodium nitrite and $0.5 \%$ purple-fleshed sweet potato powder combination, PP25 $=0.25 \%$ purple-fleshed sweet potato powder, PP50 $=0.5 \%$ purple-fleshed sweet potato powder.

a,b,c,d,e Mean values within a column followed by the same letter are not significantly different $(\mathrm{p}>0.05)$. 
had the highest chroma and hue values, respectively, and they were significantly different when compared to other sausages $(\mathrm{p}<0.05)$ Shear force and texture property measurement of Adding PFP to cooked pork sausages changed brittleness

Table 5. Shear force and texture properties of cooked pork sausages containing two different percentages of purple sweet potato combined with nitrite during 6 wks of storage at $4^{\circ} \mathrm{C}$

\begin{tabular}{|c|c|c|c|c|c|}
\hline \multirow{2}{*}{ Items } & \multirow{2}{*}{ Treatments $^{1}$} & \multicolumn{4}{|c|}{ Storage (wks) } \\
\hline & & 0 & 2 & 4 & 6 \\
\hline \multirow{5}{*}{$\begin{array}{l}\text { Shear force } \\
\left(\mathrm{kg} / \mathrm{cm}^{2}\right)\end{array}$} & $\mathrm{CON}$ & $1.37 \pm 0.02^{\mathrm{b}}$ & $1.24 \pm 0.05^{\mathrm{d}}$ & $1.17 \pm 0.02^{\mathrm{a}}$ & $1.34 \pm 0.02^{\mathrm{bc}}$ \\
\hline & SP25 & $1.35 \pm 0.03^{\mathrm{b}}$ & $1.56 \pm 0.04^{c}$ & $0.77 \pm 0.03^{\mathrm{d}}$ & $1.36 \pm 0.01^{\mathrm{ab}}$ \\
\hline & SP50 & $1.36 \pm 0.02^{\mathrm{b}}$ & $1.54 \pm 0.06^{\mathrm{c}}$ & $0.82 \pm 0.03^{\mathrm{cd}}$ & $1.28 \pm 0.01^{\mathrm{d}}$ \\
\hline & PP25 & $1.44 \pm 0.02^{\mathrm{a}}$ & $1.91 \pm 0.03^{\mathrm{a}}$ & $0.83 \pm 0.02^{\mathrm{bc}}$ & $1.37 \pm 0.02^{\mathrm{a}}$ \\
\hline & PP50 & $1.47 \pm 0.02^{\mathrm{a}}$ & $1.67 \pm 0.03^{\mathrm{b}}$ & $0.88 \pm 0.04^{b}$ & $1.33 \pm 0.02^{\mathrm{c}}$ \\
\hline \multirow{5}{*}{$\begin{array}{l}\text { Brittleness } \\
(\mathrm{kg})\end{array}$} & $\mathrm{CON}$ & $0.24 \pm 0.01^{\mathrm{d}}$ & $0.30 \pm 0.03^{b}$ & $0.33 \pm 0.02^{\mathrm{a}}$ & $0.33 \pm 0.01$ \\
\hline & SP25 & $0.29 \pm 0.01^{\mathrm{bc}}$ & $0.31 \pm 0.01^{\mathrm{b}}$ & $0.33 \pm 0.01^{\mathrm{a}}$ & $0.34 \pm 0.02$ \\
\hline & SP50 & $0.26 \pm 0.01^{\mathrm{cd}}$ & $0.31 \pm 0.01^{\mathrm{b}}$ & $0.30 \pm 0.01^{\mathrm{b}}$ & $0.32 \pm 0.01$ \\
\hline & PP25 & $0.31 \pm 0.01^{\mathrm{b}}$ & $0.35 \pm 0.00^{\mathrm{a}}$ & $0.33 \pm 0.01^{\mathrm{a}}$ & $0.33 \pm 0.02$ \\
\hline & PP50 & $0.34 \pm 0.03^{\mathrm{a}}$ & $0.35 \pm 0.01^{\mathrm{a}}$ & $0.33 \pm 0.02^{\mathrm{a}}$ & $0.34 \pm 0.02$ \\
\hline \multirow{5}{*}{$\begin{array}{l}\text { Hardness } \\
(\mathrm{kg})\end{array}$} & $\mathrm{CON}$ & $0.24 \pm 0.01^{\mathrm{ab}}$ & $0.23 \pm 0.01^{\mathrm{a}}$ & $0.25 \pm 0.01^{\mathrm{a}}$ & $0.26 \pm 0.01$ \\
\hline & SP25 & $0.23 \pm 0.02^{\mathrm{ab}}$ & $0.22 \pm 0.02^{\mathrm{ab}}$ & $0.21 \pm 0.02^{\mathrm{b}}$ & $0.25 \pm 0.02$ \\
\hline & SP50 & $0.20 \pm 0.01^{\mathrm{c}}$ & $0.20 \pm 0.02^{\mathrm{b}}$ & $0.19 \pm 0.02^{\mathrm{b}}$ & $0.23 \pm 0.01$ \\
\hline & PP25 & $0.22 \pm 0.01^{\mathrm{bc}}$ & $0.25 \pm 0.01^{\mathrm{a}}$ & $0.24 \pm 0.01^{\mathrm{a}}$ & $0.24 \pm 0.02$ \\
\hline & PP50 & $0.26 \pm 0.02^{\mathrm{a}}$ & $0.24 \pm 0.01^{\mathrm{a}}$ & $0.25 \pm 0.01^{\mathrm{a}}$ & $0.23 \pm 0.02$ \\
\hline \multirow{5}{*}{$\begin{array}{l}\text { Cohesiveness } \\
(\%)\end{array}$} & $\mathrm{CON}$ & $1.81 \pm 1.07^{\mathrm{a}}$ & $0.60 \pm 0.03$ & $0.60 \pm 0.02$ & $0.58 \pm 0.04$ \\
\hline & SP25 & $0.75 \pm 0.13^{b}$ & $0.66 \pm 0.03$ & $0.63 \pm 0.02$ & $0.61 \pm 0.02$ \\
\hline & SP50 & $0.59 \pm 0.02^{b}$ & $0.62 \pm 0.02$ & $0.62 \pm 0.02$ & $0.62 \pm 0.03$ \\
\hline & PP25 & $0.60 \pm 0.05^{\mathrm{b}}$ & $0.61 \pm 0.03$ & $0.61 \pm 0.03$ & $0.61 \pm 0.02$ \\
\hline & PP50 & $0.60 \pm 0.03^{b}$ & $0.61 \pm 0.02$ & $0.61 \pm 0.02$ & $0.59 \pm 0.02$ \\
\hline \multirow{5}{*}{$\begin{array}{l}\text { Springiness } \\
(\mathrm{mm})\end{array}$} & $\mathrm{CON}$ & $2.92 \pm 1.62^{\mathrm{a}}$ & $1.00 \pm 0.01$ & $1.00 \pm 0.00$ & $1.02 \pm 0.03$ \\
\hline & SP25 & $1.15 \pm 0.15^{\mathrm{b}}$ & $1.00 \pm 0.00$ & $1.00 \pm 0.00$ & $1.00 \pm 0.01$ \\
\hline & SP50 & $1.01 \pm 0.02^{\mathrm{b}}$ & $1.00 \pm 0.00$ & $1.01 \pm 0.01$ & $1.00 \pm 0.00$ \\
\hline & PP25 & $1.01 \pm 0.02^{\mathrm{b}}$ & $1.01 \pm 0.01$ & $1.00 \pm 0.00$ & $1.02 \pm 0.03$ \\
\hline & PP50 & $1.00 \pm 0.01^{\mathrm{b}}$ & $1.01 \pm 0.01$ & $1.01 \pm 0.01$ & $1.00 \pm 0.00$ \\
\hline \multirow{5}{*}{$\begin{array}{l}\text { Gumminess } \\
(\mathrm{kg})\end{array}$} & $\mathrm{CON}$ & $0.43 \pm 0.25$ & $0.18 \pm 0.02$ & $0.20 \pm 0.02$ & $0.19 \pm 0.01$ \\
\hline & SP25 & $0.22 \pm 0.03$ & $0.20 \pm 0.01$ & $0.20 \pm 0.01$ & $0.21 \pm 0.02$ \\
\hline & SP50 & $0.16 \pm 0.01$ & $0.20 \pm 0.01$ & $0.19 \pm 0.01$ & $0.19 \pm 0.01$ \\
\hline & PP25 & $0.19 \pm 0.02$ & $0.21 \pm 0.01$ & $0.20 \pm 0.01$ & $0.20 \pm 0.01$ \\
\hline & PP50 & $0.20 \pm 0.03$ & $0.21 \pm 0.01$ & $0.20 \pm 0.01$ & $0.20 \pm 0.01$ \\
\hline \multirow{5}{*}{$\begin{array}{c}\text { Chewiness } \\
(\mathrm{kg}, \mathrm{mm})\end{array}$} & $\mathrm{CON}$ & $1.54 \pm 1.21^{\mathrm{a}}$ & $0.18 \pm 0.02$ & $0.20 \pm 0.02$ & $0.20 \pm 0.02$ \\
\hline & SP25 & $0.25 \pm 0.07^{\mathrm{b}}$ & $0.20 \pm 0.01$ & $0.20 \pm 0.01$ & $0.21 \pm 0.02$ \\
\hline & SP50 & $0.16 \pm 0.01^{\mathrm{b}}$ & $0.20 \pm 0.01$ & $0.19 \pm 0.01$ & $0.19 \pm 0.01$ \\
\hline & PP25 & $0.19 \pm 0.02^{\mathrm{b}}$ & $0.21 \pm 0.02$ & $0.20 \pm 0.01$ & $0.21 \pm 0.01$ \\
\hline & PP50 & $0.20 \pm 0.03^{b}$ & $0.21 \pm 0.02$ & $0.20 \pm 0.01$ & $0.20 \pm 0.01$ \\
\hline \multirow[t]{5}{*}{ Adhesiveness } & $\mathrm{CON}$ & $0.10 \pm 0.02^{\mathrm{b}}$ & $0.13 \pm 0.02$ & $0.16 \pm 0.01$ & $0.15 \pm 0.02$ \\
\hline & SP25 & $0.15 \pm 0.01^{\mathrm{a}}$ & $0.15 \pm 0.01$ & $0.16 \pm 0.01$ & $0.17 \pm 0.01$ \\
\hline & SP50 & $0.14 \pm 0.01^{\mathrm{a}}$ & $0.14 \pm 0.01$ & $0.15 \pm 0.00$ & $0.16 \pm 0.01$ \\
\hline & PP25 & $0.14 \pm 0.01^{\mathrm{a}}$ & $0.16 \pm 0.00$ & $0.14 \pm 0.01$ & $0.15 \pm 0.01$ \\
\hline & PP50 & $0.15 \pm 0.02^{\mathrm{a}}$ & $0.15 \pm 0.01$ & $0.15 \pm 0.00$ & $0.16 \pm 0.01$ \\
\hline
\end{tabular}

Data represent mean \pm standard deviation.

${ }^{1}$ Treatment: CON $($ control $)=0.01 \%$ sodium nitrite, SP25 $=0.005 \%$ sodium nitrite and $0.25 \%$ purple-fleshed sweet potato powder combination, SP50 $=$ $0.005 \%$ sodium nitrite and $0.5 \%$ purple-fleshed sweet potato powder combination, PP $25=0.25 \%$ purple-fleshed sweet potato powder, PP50 $=0.5 \%$ purple-fleshed sweet potato powder.

a,b,c,d Mean values within a column followed by the same letter are not significantly different $(\mathrm{p}>0.05)$ 
and hardness. Significantly higher brittleness and hardness were observed when PFP powder was added to sausages and stored for up to 4 wks $(\mathrm{p}<0.05)$, but no difference was observed after 4 wks of storage $(p>0.05)$. Additionally, in contrast to the brittleness of sausages, the hardness of sausages containing $0.01 \%$ sodium nitrite $(\mathrm{CON})$, showed a similar trend as the PP25 and PP50 sausages $(\mathrm{p}<0.05)$.

\section{Sensory evaluation of cooked pork sausages}

A significantly favorable color score was given to samples from the CON, SP25, and SP50 groups after storage for 2 to $6 \mathrm{wks}$ (Table 6). But no significant effects were indentified even though two different amounts of PFP powder were added to the sausages $(p>0.05)$. Significant effects of aroma, flavor, and overall acceptability were observed at $6 \mathrm{wks}$ of storage, and panelists favored sausages containing both sodium nitrite and PFP powder compared to those containing PFP powder alone $(\mathrm{p}<0.05)$. Additionally, the amount of PFP powder added to sausages positively influenced flavor and springiness properties, but a difference was only observed at 6 wks of storage $(p<0.05)$.

\section{DISCUSSION}

PFP, a common sweet potato in the Republic of Korea, influenced crude protein content when it was added to pork sausages and then cooked (Table 2). Additionally, $\mathrm{pH}$ values were lower in the sausages with added PFP powder (Table

Table 6. Sensory evaluation of cooked pork sausages containing two different percentages of purple sweet potato combined with nitrite during $6 \mathrm{wks}$ of storage at $4^{\circ} \mathrm{C}$

\begin{tabular}{|c|c|c|c|c|c|}
\hline \multirow{2}{*}{ Items } & \multirow{2}{*}{ Treatments ${ }^{1}$} & \multicolumn{4}{|c|}{ Storage (wks) } \\
\hline & & 0 & 2 & 4 & 6 \\
\hline \multirow[t]{5}{*}{ Color } & $\mathrm{CON}$ & $7.63 \pm 0.93$ & $7.08 \pm 0.20^{\mathrm{a}}$ & $7.42 \pm 0.38^{\mathrm{a}}$ & $7.33 \pm 0.26^{\mathrm{a}}$ \\
\hline & SP25 & $7.00 \pm 0.71$ & $6.75 \pm 0.27^{\mathrm{ab}}$ & $7.08 \pm 0.20^{\mathrm{ab}}$ & $7.00 \pm 0.32^{\mathrm{ab}}$ \\
\hline & SP50 & $7.00 \pm 0.63$ & $6.83 \pm 0.41^{\mathrm{a}}$ & $6.75 \pm 0.27^{\mathrm{bc}}$ & $7.00 \pm 0.32^{\mathrm{ab}}$ \\
\hline & PP25 & $6.67 \pm 0.41$ & $6.08 \pm 0.38^{\mathrm{c}}$ & $6.58 \pm 0.38^{c}$ & $6.58 \pm 0.49^{b}$ \\
\hline & PP50 & $6.83 \pm 0.75$ & $6.42 \pm 0.20^{\mathrm{bc}}$ & $6.50 \pm 0.45^{\mathrm{c}}$ & $6.58 \pm 0.49^{\mathrm{b}}$ \\
\hline \multirow[t]{5}{*}{ Aroma } & $\mathrm{CON}$ & $7.08 \pm 0.49$ & $7.00 \pm 0.32$ & $7.17 \pm 0.26$ & $6.92 \pm 0.38^{\mathrm{a}}$ \\
\hline & SP25 & $6.92 \pm 0.49$ & $7.25 \pm 0.27$ & $7.17 \pm 0.26$ & $6.92 \pm 0.20^{\mathrm{a}}$ \\
\hline & SP50 & $7.17 \pm 0.61$ & $7.08 \pm 0.38$ & $7.08 \pm 0.49$ & $7.17 \pm 0.41^{\mathrm{a}}$ \\
\hline & PP25 & $7.08 \pm 0.58$ & $6.67 \pm 0.52$ & $7.17 \pm 0.26$ & $5.75 \pm 0.61^{\mathrm{b}}$ \\
\hline & PP50 & $7.00 \pm 0.63$ & $7.25 \pm 0.27$ & $7.25 \pm 0.27$ & $6.92 \pm 0.38^{\mathrm{a}}$ \\
\hline \multirow[t]{5}{*}{ Flavor } & $\mathrm{CON}$ & $7.92 \pm 0.97$ & $7.42 \pm 0.49$ & $6.50 \pm 0.45$ & $6.42 \pm 0.20^{\mathrm{ab}}$ \\
\hline & SP25 & $8.00 \pm 0.84$ & $6.83 \pm 0.26$ & $7.00 \pm 0.55$ & $6.00 \pm 0.63^{\mathrm{b}}$ \\
\hline & SP50 & $7.75 \pm 0.94$ & $6.83 \pm 0.41$ & $6.92 \pm 0.86$ & $6.75 \pm 0.52^{\mathrm{a}}$ \\
\hline & PP25 & $7.50 \pm 0.71$ & $6.92 \pm 0.49$ & $7.33 \pm 0.61$ & $5.33 \pm 0.61^{\mathrm{c}}$ \\
\hline & PP50 & $7.25 \pm 0.52$ & $7.25 \pm 0.42$ & $6.92 \pm 0.49$ & $6.25 \pm 0.27^{\mathrm{ab}}$ \\
\hline \multirow[t]{5}{*}{ Springiness } & $\mathrm{CON}$ & $7.75 \pm 0.82$ & $7.42 \pm 0.38^{\mathrm{a}}$ & $6.67 \pm 0.26$ & $6.17 \pm 0.26^{\mathrm{a}}$ \\
\hline & SP25 & $7.17 \pm 0.82$ & $6.75 \pm 0.27^{\mathrm{b}}$ & $6.42 \pm 0.66$ & $5.58 \pm 0.38^{\mathrm{b}}$ \\
\hline & SP50 & $7.08 \pm 0.58$ & $6.50 \pm 0.45^{\mathrm{b}}$ & $6.92 \pm 0.38$ & $6.08 \pm 0.38^{\mathrm{a}}$ \\
\hline & PP25 & $7.33 \pm 0.75$ & $6.75 \pm 0.42^{\mathrm{b}}$ & $7.00 \pm 0.63$ & $6.33 \pm 0.52^{\mathrm{a}}$ \\
\hline & PP50 & $7.17 \pm 0.75$ & $7.33 \pm 0.41^{\mathrm{a}}$ & $6.83 \pm 0.41$ & $6.42 \pm 0.38^{\mathrm{a}}$ \\
\hline \multirow[t]{5}{*}{ Juiciness } & $\mathrm{CON}$ & $7.92 \pm 0.58$ & $7.33 \pm 0.26$ & $7.08 \pm 0.49$ & $6.92 \pm 0.80$ \\
\hline & SP25 & $7.67 \pm 0.75$ & $6.83 \pm 0.41$ & $6.83 \pm 0.52$ & $6.67 \pm 0.82$ \\
\hline & SP50 & $7.42 \pm 0.97$ & $7.00 \pm 0.45$ & $7.17 \pm 0.52$ & $6.92 \pm 0.49$ \\
\hline & PP25 & $7.25 \pm 0.69$ & $6.75 \pm 0.42$ & $7.33 \pm 0.26$ & $6.42 \pm 0.49$ \\
\hline & PP50 & $7.17 \pm 0.41$ & $7.08 \pm 0.20$ & $7.00 \pm 0.45$ & $6.67 \pm 0.41$ \\
\hline \multirow[t]{5}{*}{ Overall acceptability } & $\mathrm{CON}$ & $7.83 \pm 0.52$ & $7.42 \pm 0.38^{\mathrm{a}}$ & $6.58 \pm 0.38$ & $6.67 \pm 0.26^{\mathrm{a}}$ \\
\hline & SP25 & $7.67 \pm 0.75$ & $7.00 \pm 0.00^{\mathrm{b}}$ & $7.00 \pm 0.32$ & $6.08 \pm 0.58^{\mathrm{a}}$ \\
\hline & SP50 & $7.42 \pm 0.74$ & $6.83 \pm 0.26^{\mathrm{bc}}$ & $6.83 \pm 0.68$ & $6.50 \pm 0.45^{\mathrm{a}}$ \\
\hline & PP25 & $7.25 \pm 0.82$ & $6.58 \pm 0.38^{\mathrm{c}}$ & $7.08 \pm 0.58$ & $5.33 \pm 0.75^{\mathrm{b}}$ \\
\hline & PP50 & $7.17 \pm 0.52$ & $7.08 \pm 0.20^{\mathrm{b}}$ & $6.83 \pm 0.52$ & $6.25 \pm 0.27^{\mathrm{a}}$ \\
\hline
\end{tabular}

Data represent mean \pm standard deviation.

${ }^{1}$ Treatment: CON (control) $=0.01 \%$ sodium nitrite, SP25 $=0.005 \%$ sodium nitrite and $0.25 \%$ purple-fleshed sweet potato powder combination, SP50 $=$ $0.005 \%$ sodium nitrite and $0.5 \%$ purple-fleshed sweet potato powder combination, PP25 $=0.25 \%$ purple-fleshed sweet potato powder, PP50 $=0.5 \%$ purple-fleshed sweet potato powder.

${ }^{a, b, c}$ Mean values within a column followed by the same letter are not significantly different $(\mathrm{p}>0.05)$. 
3). The lowest $\mathrm{pH}$ value was confirmed for the $0.5 \%$ added PFP powder sausages at 6 wks of storage. As expected, adding PFP powder to sausages resulted in a low $\mathrm{pH}$ value compared to that of the control and may have impacted the positive effects on residual nitrite. Marco et al. (2006) noted that nitrite content in sausages quickly declines due to the high reactivity of nitrite as compared to that of sausages containing nitrate and such a reaction may be accelerated due to components added to sausages. Therefore, $\mathrm{pH}$, an effective internal factor, may influence the reactivity of nitrite, resulting in low nitrite residues in sausages during storage.

As shown in Table 4, the CIE $\mathrm{L}^{*}$ and whiteness (W) showed high values in sausages sampled from the control, and both the CIE $\mathrm{L}^{*}$ and $\mathrm{W}$ values were negatively affected by adding PFP powder. The $\mathrm{pH}$ values of sausages containing PFP powder were low enough to release water, indicating possible light dispersion during sausage color measurements (Qiao et al., 2001; Huff-Lonergan and Lonergan, 2005). However, $\mathrm{pH}$ was not the only factor influencing lightness and whiteness of the sausages. The cyanidin and peonidin pigments extracted from PFP may alter the color of cooked pork sausages and may produce a slightly yellow color (Hagiwara et al., 2002; Suda et al., 2003; Mozetič et al., 2004). Therefore, sausages in the PP25 and PP50 treatments had higher CIE b* values compared to those of the other treatments, but the CIE $b^{*}$ value did not increase dose dependently.

Adding PFP powder tended to increase the shear force and brittleness of cooked pork sausages for up to 6 wks of storage. This occurred because PFP powder contains starch, and the molecular network within the starch granules was affected by heat during cooking (González and Pérez, 2002; Lai et al., 2003). Unexpectedly, the PFP powder did not influence the hardness of cooked pork sausages. It seemed that cooked pork sausages were firm due to the $\mathrm{pH}$ value of 6.0. Wang et al. (2000) indicated that the viscosity of rice flour was the highest when $\mathrm{pH}$ reached 6.2, whereas $\mathrm{pH} 4.1$ reduced rice flour viscosity. The $\mathrm{pH}$ values of sausages in the PP25 and PP50 treatments were 5.74 to 6.17 , and they may have influenced the viscosity of the starch in the PFP. However, such an effect was not fully determined in SP25 and SP50 sausages containing the sodium nitrite and PFP powder combination. As the sodium nitrite was provided as a $\mathrm{NaNO}_{2}$ to sausages, $\mathrm{Na}$ ions or other anions lowered or delayed the starch viscosity and/or gelatinization, making the sausages firmer (Nutting, 1952; Krog, 1973; Kim et al., 1987). Thus, the combination of sodium nitrite and PFP resulted in lower hardness values of sausages in the PP25 and PP50 treatments.

Overall effects on color and textural characteristics of PFP in the sensory evaluation were measured, and objective color scores of sausages were influenced. The effects of the PFP powder on objective color were due to $\mathrm{pH}$, pigments from the PFP powder, and the formation of nitrosohemochrome, which provided a bright pink-red color due to cooking ( $\mathrm{Lu}, 2010)$. Objective color scores were better in sausages with added sodium nitrite and PFP powder as compared to those of sausages containing sodium nitrite only. In contrast to the springiness result in Table 5, sausages from the CON, SP50, PP25 and PP50 treatments had significantly different springiness values compared to those of SP25 sausages. Therefore, the overall acceptability of sausages was best when sodium nitrite and/or PFP powder was added alone or in combination to pork sausages except sausages sampled from the SP25 treatment.

In conclusion, adding PFP seemed to provide acceptable effects for subjective and objective color, and textural properties tended to improve as compared to those of sausages containing PFP alone. However, the ratio or concentration of PFP in combination with sodium nitrite must be determined to provide better textural and sensory properties.

\section{ACKNOWLEDGEMENTS}

This study was sponsored by Priority Research Centers Program through the National Research Foundation of Korea (FRF), funded by the Ministry of Education, Science, and Technology (2009-0093813).

\section{REFERENCES}

Ahn, H. J., J. H. Kim, C. Jo, C. H. Lee and M. W. Hyun. 2002. Reduction of carcinogenic N-nitrosamines and residual nitrite in model system sausage by irradiation. J. Food Sci. 67:1370-1373.

AMSA. 1995. Research guidelines for cookery, sensory evaluation and tenderness measurements of fresh meat. Am. Meat Sci. Assoc., Chicago, IL, USA.

AOAC. 1990. Official methods of analysis. $15^{\text {th }}$ ed., Association of Official Analytical Chemists, Washington DC, USA.

Bozkurt, H. and O. Erkmen. 2004. Effect of nitrate/nitrite on the quality of sausage (sucuk) during ripening and storage. J. Sci. Food Agric. 84:279-286.

Cevallos-Casals, B. A. and L. Cisneros-Zevallos. 2004. Stability of anthocyanin-based aqueous extracts of Andean purple corn and red-fleshed sweet potato compared to synthetic and natural colorants. Food Chem. 86:69-77.

González, Z. and E. Pérez. 2002. Effect of acetylation on some properties of rice starch. Starch. 54:148-154.

Hagiwara, A., H. Yoshino, T. Ichihara, M. Kawabe, S. Tamano, H. Aoki, T. Koda, M. Nakamura, K. Imaida, N. Ito and T. Shirai. 2002. Prevention by natural food anthocyanins, purple sweet potato color and red cabbage color, of 2-amino-1-methyl-6- 
phenylimidazo[4,5-b] pyridine (PhIP)-associated colorectal carcinogenesis in rats initiated with 1,2-dimethylhydrazine. J. Toxicol. Sci. 27:57-68.

Huff-Lonergan, E. and S. M. Lonergan. 2005. Mechanisms of water-holding capacity of meat: The role of postmortem biochemical and structural changes. Meat Sci. 71:94-204.

Islam, S., M. Yoshimoto, N. Terahara and O. Yamakawa. 2002. Anthocyanin compositions in sweetpotato (Ipomoea batatas L.) leaves. Biosci. Biotechnol. Biochem. 66:2483-2486.

Jo, C., H. J. Ahn, J. H. Son, J. W. Lee and M. W. Byun. 2003. Packaging and irradiation effect on lipid oxidation, color, residual nitrite content, and nitrosamine formation in cooked pork sausage. Food Control 14:7-12.

Kano, M., T. Takayanagi, K. Harada, K. Makino and F. Ishikawa 2005. Antioxidative activity of anthocyanins from purple sweet patato, Ipomoera batatas cultivar Ayamurasaki. Biosci. Biotechnol. Biochem. 69:979-988.

Kealy, T. 2006. Application of liquid and solid rheological technologies to the textureal characterization of semi-solid foods. Food Res. Int. 39:265-276.

Kim, I-H., S-G. Kim and S-Y. Lee. 1987. Effect of phosphate on rheological properties of gelatinized rice starch solution. Korean J. Food Sci. Technol. 19:366-370.

Krog, N. 1973. Influence of food emulsifiers on pasting temperature and viscosity of various starches. Starch-Stärke 25:22-27.

Lai, L-S., Y-L. Liu and P-H. Lin. 2003. Pheological/textural properties of starch and crude hsian-tsao leaf gum mixed systems. J. Sci. Food Agric. 83:1051-1058.

Lee, S-C., S-Y. Kim, S-M. Jeong and J-H. Park. 2006. Effect of far-infrared irradiation on catechins and nitrite scavenging activity of green tea. J. Agric. Food Chem. 54:399-403.

$\mathrm{Lu}$, Y. 2010. The development of a cured, fermented sheep meat sausage designed to minimize species and pastoral-diet flavours. Master Thesis, AUT University, New Zealand. p.7.
Marco, A., J. L. Navarro and M. Flores. 2006. The influence of nitrite and nitrate on microbial, chemical and sensory parameters of slow dry fermented sausage. Meat Sci. 73:660673

Meilgaard, M., G. V. Civille and B. T. Carr. 1991. Sensory evaluation techniques (2nd ed.). CRC Press, Boca Raton, FL. USA.

Mozetič, B., P. Trebše, M. Simčič and J. Hribar. 2004. Changes of anthocyanins and hydroxycinnamic acids affecting the skin colour during maturation of sweet cherries (Prunus avium L.). LWT-Food Sci. Technol. 37:123-128.

Nutting, G. C. 1952. Effect of electrolytes on the viscosity of potato starch pastes. J. Colloid Sci. 7:128-139.

Qiao, M., D. L. Fletcher, D. P. Smith and J. K. Northcutt. 2001. The effect of broiler breast meat color on $\mathrm{pH}$, moisture, waterholding capacity, and emulsification capacity. Poult. Sci. 80:676-680.

Pearson, D. 1976. The chemical analysis of foods (7th ed.). Churchill, Livingston. p. 386.

Rundlöf, T., E. Olsson, A. Wiernik, S. Back, M. Aune, L. Johansson and I. Wahlberg. 2000. Potential nitrite scavengers as inhibitors of the formation of $\mathrm{N}$-nitrosamines in solution and tobacco matrix systems. J. Agric. Food Chem. 48:43814388 .

SAS. 1998. SAS/STAT software for PC. Release 6.12, SAS Institute Inc., Cary, NC. USA.

Suda, I., T. Oki, M. Masuda, M. Kobayashi, Y. Nishiba and S. Furuta. 2003. Physiological functionality of purple-fleshed sweet potatoes containing anthocyanins and their utilization in foods. Japan Agric. Res. Quart. 37:167-173.

Teow, C. C., V-D. Truong, R. F. McFeeters, R. L. Thompson, K. V. Pecota and G. C. Yencho. 2007. Antioxidant activities, phenolic and B-carotene contents of sweet potato genotypes wit varying flesh colours. Food Chem. 103:829-838.

Wang, H-H., D-W. Sun, Q. Zeng and Y. Lu. 2000. Effect of pH, corn starch and phosphates on the pasting properties of rice flour. J. Food Eng. 46:133-138. 